Journal of the Brazilian Society of Mechanical Sciences and Engineering

Publisher: Springer

Impact Factor: 1.235

ISSN: 1678-5878 (print version)

ISSN: 1806-3691 (electronic version)

Accepted July $27^{\text {th }} 2017$

\title{
INFLUENCE OF STEFAN BLOWING ON NANOFLUID FLOW SUBMERGED IN MICROORGANISMS WITH LEADING EDGE ACCRETION OR ABLATION
}

\author{
Md Faisal Md Basir ${ }^{*}$, M.J. Uddin ${ }^{2}$, and O. Anwar Bég ${ }^{\mathbf{3}}$ and A. I. Md. Ismail ${ }^{\mathbf{1}}$ \\ ${ }^{1}$ School of Mathematical Sciences, University Sains Malaysia,11800, Penang, Malaysia. \\ ${ }^{2}$ American International University-Bangladesh, Banani, Dhaka 1213, Bangladesh. \\ ${ }^{3}$ Aeronautical/Mechanical Engineering, University of Salford, Manchester, M54WT, UK. \\ *Corresponding author: faisalbasir91@gmail.com, \\ Co-author emails: ${ }^{2}$ drjashim@aiub.edu, ${ }^{3}$ ahmad_izani@usm.my, \\ 33.A.Beg@salford.ac.uk
}

\begin{abstract}
The unsteady forced convective boundary layer flow of viscous incompressible fluid containing both nanoparticles and gyrotactic microorganisms, from a flat surface with leading edge accretion (or ablation), is investigated theoretically. Utilizing appropriate similarity transformations for the velocity, temperature, nanoparticle volume fraction and motile microorganism density, the governing conservation equations are rendered into a system of coupled, nonlinear, similarity ordinary differential equations. These equations, subjected to imposed boundary conditions, are solved numerically using the Runge-KuttaFehlberg fourth-fifth order numerical method in the MAPLE symbolic software. Good agreement between our computations and previous solutions is achieved. The effect of selected parameters on flow velocity, temperature, nano-particle volume fraction (concentration) and motile microorganism density function is investigated. Furthermore, tabular solutions are included for skin friction, wall heat transfer rate, nano-particle mass transfer rate and microorganism transfer rate. Applications of the study arise in advanced micro-flow devices to assess nanoparticle toxicity.
\end{abstract}

Keywords: Bioconvection; nanofluids; Stefan blowing; numerical solution; accretion/ablation. 


\section{NOMENCLATURE}

chemotaxis constant $(m)$

C

nano-particles volume fraction $(-)$

$C_{w}$

wall nano-particle volume fraction $(-)$

$C_{\infty}$

ambient nano-particle volume fraction $(-)$

$C_{f_{\bar{x}}}$

local skin friction coefficient along the $\bar{x}(-)$

$c_{p}$

specific heat at constant pressure $\left(\frac{\mathrm{J}}{\mathrm{kgK}}\right)$

$D_{B}$

Brownian diffusion coefficient $\left(\frac{m^{2}}{s}\right)$

$D_{n}$

microorganism diffusion coefficient $\left(\frac{m^{2}}{s}\right)$

$D_{T}$

thermophoresis diffusion coefficient $\left(\frac{m^{2}}{s}\right)$

$f(\eta)$

dimensionless stream function $(-)$

$\vec{j}$

vector flux of micro-organisms $\left(\frac{\mathrm{kg}}{\mathrm{m}^{2} \mathrm{~s}}\right)$

$k$

thermal conductivity $\left(\frac{W}{m K}\right)$

$L b$

bioconvection Lewis number $\left(L b=\frac{\alpha}{D_{n}}\right)(-)$

Le

Lewis number $\left(L e=\frac{\alpha}{D_{B}}\right)(-)$

$\mathrm{Nb}$

Brownian motion parameter $\left(N b=\frac{\tau D_{B}\left(C_{w}-C_{\infty}\right)}{\alpha}\right)(-)$

$N n_{\bar{x}} \quad$ local density number of motile microorganisms (-) 


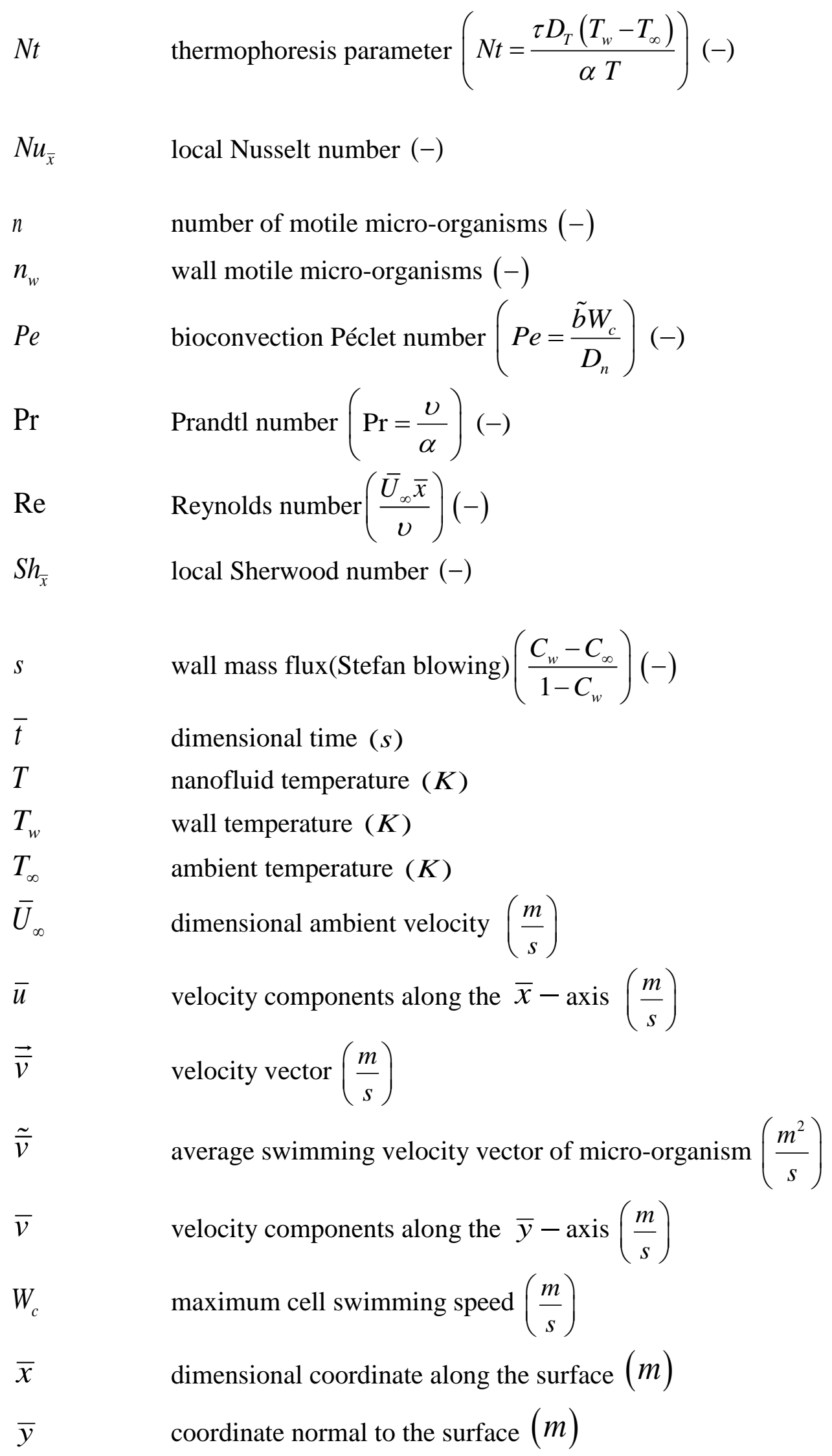




\section{Greek letters}

\begin{tabular}{|c|c|}
\hline$\alpha$ & effective thermal diffusivity $\left(\frac{m^{2}}{s}\right)$ \\
\hline$\gamma$ & leading edge accretion/ablation $(-)$ \\
\hline$\eta$ & independent similarity variable $(-)$ \\
\hline$\theta(\eta)$ & dimensionless temperature $(-)$ \\
\hline$\mu$ & dynamic viscosity $\left(\frac{k g}{m s}\right)$ \\
\hline$U$ & kinematic viscosity $\left(\frac{m^{2}}{s}\right)$ \\
\hline$\rho$ & fluid density $\left(\frac{\mathrm{kg}}{\mathrm{m}^{3}}\right)$ \\
\hline$\pi$ & pi $(-)$ \\
\hline$(\rho c)_{f}$ & volumetric heat capacity of the fluid $\left(\frac{J}{m^{3} K}\right)$ \\
\hline$(\rho c)_{p}$ & volumetric heat capacity of the nanoparticle material $\left(\frac{J}{m^{3} K}\right)$ \\
\hline$\sigma$ & dimensionless time variable $\left(\bar{U}_{\infty} \bar{t} / \bar{x}\right)(-)$ \\
\hline$\tau$ & $\begin{array}{l}\text { ratio of the effective heat capacity of the nanoparticle material to the fluid } \\
\text { heat capacity }\left(\frac{(\rho c)_{p}}{(\rho c)_{f}}\right)(-)\end{array}$ \\
\hline$\phi(\eta)$ & dimensionless nanoparticles volume fraction $(-)$ \\
\hline$\chi(\eta)$ & dimensionless number of motile micro-organisms $(-)$ \\
\hline$\psi$ & streamline function $(-)$ \\
\hline
\end{tabular}

\section{Subscripts}

( )'

ordinary differentiation with respect to $\eta$

()$_{w}$ condition at wall

()$_{\infty}$ condition in free stream 


\section{INTRODUCTION}

The analysis of convective heat and mass transfer with nanoparticles has gained an abundance of attention amongst researchers in recent years. Heat exchange may be enhanced by adding nanoparticles with high thermal properties in low volume fraction within the liquid that leads to modern class of fluids known as nanofluids [1]. Conventional heat transfer fluids, for example, water, oil and ethylene glycol are known to be poor heat transfer fluids [2]. As the thermal conductivity of these liquids assumes a critical part in the heat transfer coefficient, therefore various strategies have been explored to upgrade the thermal conductivity of these liquids. It is now established that a significant improvement in thermal conductivity can be achieved by including nanometersized particles suspended in conventional heat transfer liquids. In this manner, nanomaterials are perceived to be more successful in smaller scale/nano electromechanical devices, advanced cooling frameworks, extensive scale thermal frameworks in evaporators, heat exchangers and mechanical cooling applications. Nanofluids are generally stable under a variety of operation conditions with no additional issues of disintegration, sedimentation, clogging, coagulation or extra weight drop. This is a direct result of the small size and low volume nano-particles required for thermal conductivity improvement [3]. The base fluid, or dissolving medium, can be aqueous or non-aqueous in nature and nanoparticles may comprise metals, carbides, oxides, carbon 
nanotubes or nitrides. Nanoparticles shapes may be disks, spheres, cylindrical rods etc. [4]. Recently, many studies of computational modelling of nanofluids have been communicated with diverse applications [5-17].

Bioconvection has important applications in bio-microsystems where it is utilized, for example, to enhance mass transport as well as to enhancement and mixing. Nanofluid bioconvection occurs when the spontaneous pattern formation and density stratification is caused by the simultaneous interaction of the denser self-propelled microorganisms, nanoparticles, and buoyancy forces [18]. Microorganisms are known to be respond to certain stimuli by tending to swim in certain directions. These responses are called taxes and examples are gravitaxis, gyrotaxis, phototaxis, magneto-taxis and chemotaxis [19]. Gravitaxis refers to the swimming opposite to gravity and gyrotaxis is the swimming determined the equilibrium of torques due to viscous forces from shear flows and gravity. Phototaxis is due to the movement toward or away from light [20]. Fundamentally, the swimming of microorganisms that causes the fluid to convect increases the density of the surrounding fluid. Analytical studies of nanofluid bioconvection were first presented by [21-27]. Makinde and Animasaun [28] reported heat and mass transfer behaviour decreases the diffusion of motile microorganisms. Recently, Akbar and Khan [29] investigated the effects of magneto-bioconvection, Brownian motion and thermophoresis on free convection flow over a stretching sheet. Amirsom et al. [30] analyzed the threedimensional stagnation point flow of fluid containing both nanoparticles and gyrotactic micro-organisms with variable transport properties. Babu and Sandeep [31] simulated the non-aligned bioconvective stagnation point flow of a nanofluid comprising gyrotactic 
micro-organisms from a stretching sheet by considering nonlinear radiation and variable viscosity for both cases of oblique flow and free stream flow. Raees et al. [32] considered theoretically three-dimensional stagnation flow on a plate with anisotropic slip in a suspension of microorganisms and nanoparticles.

Boundary conditions are important in simulating convective nanofluid flows. The blowing effect comes from the concept of the Stefan problem for species transfer [33]. In practical applications, such as paper drying processes, mass transfer is achieved by evaporation [34]. The diffusion of the species produces a bulk motion of fluid and induces extra motion of the fluid [35]. In this paper, the solid surface which is affected by the blowing is not considered to be porous. Further the blowing is assumed to be due to flux transfer of species from the solid surface to outside/inside of the boundary layer. Species transfer varies on the flow field and the flow field is affected by the mass blowing at the wall. Fang and Jing [33] and Uddin et al. [36] studied the boundary layer flow considering Stefan blowing effects and verified that the blowing velocity was proportional to the mass transfer flux.

In this present paper, we have employed the Buongiorno nanofluid model [37] which incorporates both thermophoretic and Brownian motion effects. The model has been successfully deployed by several authors [38-49].

A new unsteady boundary layer model which involves a moving leading edge exhibiting a certain rate of accretion or ablation and was first proposed by Todd [50] has also stimulated considerable interest in recent years. Different velocity variation trends arise 
for different leading edge accretion/ablation effects; these can modify heat, and mass transfer rates in boundary layer flows. Different families of solutions can also arise for unsteady boundary layer flows with accretion/ablation. Further analysis of the momentum and thermal boundary layer flows with accretion and ablation effects has been conducted by [51] and [2]. Recently, Rosca and Pop [52] have considered momentum, thermal and solutal boundary layer flows using the Buongiorno nanofluid model with accretion/ablation effects. The aim of this paper is to extend the work of the [2],[50-52] to the unsteady boundary layer flow of Buongiorno nanofluid by incorporating bioconvection phenomena i.e. propulsion of motile microorganisms. The model developed is relevant to a variety of nano-biophysical fluidic devices including fuel cells, bio-modified nanomaterials processing etc.

\section{MATHEMATICAL MODEL}

We consider two-dimensional, unsteady, incompressible, viscous, constant transport property, laminar forced convective boundary layer flow of a nanofluid over a solid stationary semi-infinite plate with leading edge accretion ablation. The nanofluid contains gyrotactic microorganisms. The effects of Stefan blowing are taken into account. Let the free stream velocity be $\bar{U}_{\infty}$, the free stream temperature be $T_{\infty}$, the free stream nanoparticle volume flux as $C_{\infty}$ and the free stream microorganism be zero for $\operatorname{simplicity}\left(n_{\infty}=0\right)$. It also assumed that the uniform temperature, nanoparticle volume fraction and motile microorganism density at the plate are $T_{w}, C_{w}$ and $n_{w}$, respectively. Let $(\bar{u}, \bar{v})$ and $(\bar{x}, \bar{y})$ be the dimensional velocity components in the vertically upward 
direction (parallel to the free stream) and perpendicular to the plate, respectively. The physical configuration of the problem is visualized in Figure 1, wherein (i) represents the momentum and (ii) symbolize thermal, mass diffusion and microorganism boundary layers. Under above assumptions and following model equations proposed by [37] and [53], the vector field equations are:

$\nabla \cdot \overrightarrow{\vec{v}}=0$

$\frac{\partial \overrightarrow{\bar{v}}}{\partial \bar{t}}+(\overrightarrow{\bar{v}} \cdot \nabla) \overrightarrow{\bar{v}}=-\frac{1}{\rho} \nabla p+v \nabla^{2} \overrightarrow{\bar{v}}$

$\frac{\partial T}{\partial \bar{t}}+(\vec{v} \cdot \nabla) T=\alpha \nabla^{2} T+\tau\left[D_{B} \nabla T \cdot \nabla C+\left(\frac{D_{T}}{T_{\infty}}\right) \nabla T \cdot \nabla T\right]$

$\frac{\partial C}{\partial \bar{t}}+(\overrightarrow{\bar{v}} \cdot \nabla) C=D_{B}\left(\nabla^{2} C\right)+\left(\frac{D_{T}}{T_{\infty}}\right) \nabla^{2} T$,

$\frac{\partial n}{\partial \bar{t}}+\nabla \cdot \vec{j}=0$

where $\nabla=\frac{\partial}{\partial x} \vec{i}+\frac{\partial}{\partial y} \vec{j}, \nabla^{2}$ : Laplacian operator, $v$ : kinematic viscosity, $\rho$ : fluid density, $\alpha$ : thermal diffusivity of the fluid, $\tau=(\rho c)_{p} /(\rho c)_{f}$ : ratio of effective heat capacity of the nanoparticle material to the fluid heat capacity, $D_{B}$ : Brownian diffusion coefficient, $D_{T}$ : thermophoretic diffusion coefficient and $\vec{j}$ : flux of microorganisms that is defined as:

$\vec{j}=n \overrightarrow{\bar{v}}+n \tilde{\bar{v}}-D_{n} \nabla n$, 
In the Eq. (5), $D_{n}$ : diffusivity of microorganisms, $\tilde{\bar{v}}=\left(\frac{\tilde{b} W_{c}}{\Delta C}\right) \nabla C$ : average swimming speed velocity vector of the gyrotactic micro-organism, $\tilde{b}$ : chemotaxis constant and $W_{c}$ is the maximum cell swimming speed.

After applying boundary layer approximations or order of magnitude analysis, the Eqs. (1) - (5) can be written in scalar form as:

$$
\frac{\partial \bar{u}}{\partial \bar{x}}+\frac{\partial \bar{v}}{\partial \bar{y}}=0
$$

$\frac{\partial \bar{u}}{\partial \bar{t}}+\bar{u} \frac{\partial \bar{u}}{\partial \bar{x}}+\bar{v} \frac{\partial \bar{u}}{\partial \bar{y}}=v \frac{\partial^{2} \bar{u}}{\partial \bar{y}^{2}}+\bar{u}_{e} \frac{\partial \bar{u}_{e}}{\partial \bar{x}}+\frac{\partial \bar{u}_{e}}{\partial \bar{t}}$

$\frac{\partial T}{\partial \bar{t}}+\bar{u} \frac{\partial T}{\partial \bar{x}}+\bar{v} \frac{\partial T}{\partial \bar{y}}=\alpha \frac{\partial^{2} T}{\partial \bar{y}^{2}}+\tau D_{B} \frac{\partial T}{\partial \bar{y}} \frac{\partial C}{\partial \bar{y}}+\tau \frac{D_{T}}{T_{\infty}}\left(\frac{\partial T}{\partial \bar{y}}\right)^{2}$

$\frac{\partial C}{\partial \bar{t}}+\bar{u} \frac{\partial C}{\partial \bar{x}}+\bar{v} \frac{\partial C}{\partial \bar{y}}=D_{B} \frac{\partial^{2} C}{\partial \bar{y}^{2}}+\frac{D_{T}}{T_{\infty}} \frac{\partial^{2} T}{\partial \bar{y}^{2}}$,

$\frac{\partial n}{\partial \bar{t}}+\bar{u} \frac{\partial n}{\partial \bar{x}}+\bar{v} \frac{\partial n}{\partial \bar{y}}+\frac{\tilde{b} W_{c}}{C_{w}-C_{\infty}}\left[\frac{\partial}{\partial \bar{y}}\left(n \frac{\partial C}{\partial \bar{y}}\right)\right]=D_{n}\left(\frac{\partial^{2} n}{\partial \bar{y}^{2}}\right)$

subject to the following boundary conditions [36]:

$$
\begin{aligned}
& \bar{u}=0, \bar{v}=-\frac{D_{B}}{\left(1-C_{w}\right)}\left(\frac{\partial C}{\partial \bar{y}}\right), T=T_{w}, C=C_{w}, n=n_{w} \text { as } \bar{y}=0, \\
& \bar{u}=\bar{u}_{e}=\bar{U}_{\infty}, \quad T \rightarrow T_{\infty}, C \rightarrow C_{\infty}, n \rightarrow 0 \quad \text { as } \bar{y} \rightarrow \infty,
\end{aligned}
$$

where the following notation applies- $\overline{\boldsymbol{u}}_{e}$ : external velocity and $v$ : kinematic viscosity, $\rho$ : fluid density. 
We implement the following modified dimensional stream function, which incorporates ablation/accretion effects at the boundary layer leading edge:

$$
\psi(\bar{x}, \bar{y}, \bar{t})=\bar{U}_{\infty} \sqrt{v \bar{t} \cos \gamma+\left(v \bar{x} / \bar{U}_{\infty}\right) \sin \gamma} f(\eta)
$$

Defining the similarity variable:

$$
\eta=\bar{y} / \sqrt{v \bar{t} \cos \gamma+\left(v \bar{x} / \bar{U}_{\infty}\right) \sin \gamma}
$$

Non-dimensional temperature, nanoparticle volume fraction and microorganism density functions may be defined as follows:

$$
\theta(\eta)=\left(T-T_{\infty}\right) /\left(T_{w}-T_{\infty}\right), \quad \phi(\eta)=\left(C-C_{\infty}\right) /\left(C_{w}-C_{\infty}\right), \quad \chi(\eta)=n / n_{w} .
$$

Here the dimensionless variables are $\eta$ (similarity), $f^{\prime}(\eta)$ (velocity), $\theta(\eta)$ (temperature), $\phi(\eta)$ (nanoparticle volume fraction), $\chi(\eta)$ (microorganisms), $\gamma$ is the leading edge accretion/ablation parameter and $\bar{t}$ is the dimensional time. Subscripts $w$ and $\infty$ denote at the wall and in the free stream, respectively. Prime denotes ordinary differentiation with respect to $\eta$. The quantity $\left(v \bar{t} \cos \gamma+\left(v \bar{x} / \bar{U}_{\infty}\right) \sin \gamma\right)$ must be positive [50]. $\psi(\bar{x}, \bar{y}, \bar{t})$ is the streamline function, which defined as

$\bar{u}=\partial \psi / \partial \bar{y}$ and $\bar{v}=-\partial \psi / \partial \bar{x}$.

Substituting Eqns. (13) and (14) into Eq. (16), we obtain $\bar{u}$ and $\bar{v}$ as follows:

$$
\bar{u}=\bar{U}_{\infty} f^{\prime}, \quad \bar{v}=\frac{v}{2}\left(\eta f^{\prime}-f\right) \sin \gamma / \sqrt{v \bar{t} \cos \gamma+\left(v \bar{x} / \bar{U}_{\infty}\right) \sin \gamma}
$$

Proceeding with the analysis, the primitive partial differential conservation equations Eqns. (8)-(11) may be transformed into the following system of coupled nonlinear, similarity ordinary differential equations as follows: 


$$
\begin{aligned}
& f^{\prime \prime \prime}+\frac{1}{2}(\sin \gamma) f f^{\prime \prime}+\frac{1}{2}(\cos \gamma) \eta f^{\prime \prime}=0, \\
& \theta^{\prime \prime}+\frac{1}{2} \operatorname{Pr}(\eta \cos \gamma+f \sin \gamma) \theta^{\prime}+N b \theta^{\prime} \phi^{\prime}+N t \theta^{\prime 2}=0, \\
& \phi^{\prime \prime}+\frac{1}{2} L e \operatorname{Pr}(\eta \cos \gamma+f \sin \gamma) \phi^{\prime}+\frac{N t}{N b} \theta^{\prime \prime}=0, \\
& \chi^{\prime \prime}+\frac{1}{2} \operatorname{Pr} L b(\eta \cos \gamma+f \sin \gamma) \chi^{\prime}-P e\left[\chi \phi^{\prime \prime}+\phi^{\prime} \chi^{\prime}\right]=0,
\end{aligned}
$$

The transformed associated boundary conditions emerge as follows:

$$
\begin{aligned}
& f(0)=\frac{2}{\operatorname{Pr} L e} \frac{1}{\sin \gamma} s \phi^{\prime}, f^{\prime}(0)=0, \quad \theta(0)=1, \phi(0)=1, \\
& \chi(0)=1, f^{\prime}(\infty)=1, \quad \theta(\infty)=\phi(\infty)=\chi(\infty)=0,
\end{aligned}
$$

where the following dimensionless parameters arise: Prandtl number $(P r)$, Lewis number (Le), Brownian motion parameter $(\mathrm{Nb})$, thermophoresis parameter $(\mathrm{Nt})$, bioconvection Lewisnumber $(L b)$, bioconvection Péclet number $(P e)$ and the mass blowing/suction parameter(Stefan blowing $)(s)$. These parameters are defined respectively, as:

$$
\begin{aligned}
& \operatorname{Pr}=v / \alpha, N b=\tau D_{B}\left(C_{w}-C_{\infty}\right) / \alpha, L e=\alpha / D_{B}, N t=\tau D_{T}\left(T_{w}-T_{\infty}\right) / \alpha T_{\infty}, \\
& L b=\alpha / D_{n}, P e=\tilde{b} W_{c} / D_{n}, s=\left(C_{w}-C_{\infty}\right) /\left(1-C_{w}\right)
\end{aligned}
$$

\section{PHYSICAL QUANTITIES}

In practical applications, the gradients of the velocity, temperature, nano-particle species concentration and microorganism density function are required. These take the form of the local skin friction coefficient $C_{f_{\bar{x}}}$, the local Nusselt number $N u_{\bar{x}}$, the local Sherwood number $S h_{\bar{x}}$ and the local density number of motile microorganisms $N n_{\bar{x}}$, which may be defined thus: 


$$
\begin{aligned}
& C_{f_{\bar{x}}}=\frac{\mu}{\rho \bar{U}_{\infty}^{2}}\left(\frac{\partial \bar{u}}{\partial \bar{y}}\right)_{\bar{y}=0}, N u_{\bar{x}}=\frac{-\bar{x}}{\left(T_{w}-T_{\infty}\right)}\left(\frac{\partial T}{\partial \bar{y}}\right)_{\bar{y}=0}, \\
& S h_{\bar{x}}=\frac{-\bar{x}}{\left(C_{w}-C_{\infty}\right)}\left(\frac{\partial C}{\partial \bar{y}}\right)_{\bar{y}=0}, N n_{\bar{x}}=\frac{-\bar{x}}{n_{w}}\left(\frac{\partial n}{\partial \bar{y}}\right)_{\bar{y}=0},
\end{aligned}
$$

Employing Eqns. (13)-(17) and (24), the parameters may be re-formulated in terms of the similarity variables, as follows:

$$
\begin{aligned}
& \operatorname{Re}_{\bar{x}}^{1 / 2} C_{f_{\bar{x}}} \sqrt{\sigma \cos \gamma+\sin \gamma}=f^{\prime \prime}(0), \\
& \operatorname{Re}_{\bar{x}}^{-1 / 2} N u_{\bar{x}} \sqrt{\sigma \cos \gamma+\sin \gamma}=-\theta^{\prime}(0), \\
& \operatorname{Re}_{\bar{x}}^{-1 / 2} S h_{\bar{x}} \sqrt{\sigma \cos \gamma+\sin \gamma}=-\phi^{\prime}(0) \\
& \operatorname{Re}_{\bar{x}}^{-1 / 2} N n_{\bar{x}} \sqrt{\sigma \cos \gamma+\sin \gamma}=-\chi^{\prime}(0) .
\end{aligned}
$$

Here, $\operatorname{Re}_{\bar{x}}=\bar{U}_{\infty} \bar{x} / v$ is the local Reynolds number and $\sigma=\bar{U}_{\infty} \bar{t} / \bar{x}$ denotes dimensionless time variable, introduced by [54].

It is interesting to note that in the absence of the Eqns. (19)-(21), $s=0$ (no suction/injection), the present model reduces to [50]. It is also noteworthy that $s>0$ for species transfer from the wall to the free stream (evaporation), while $s<0$ for species transfer from the free stream to the wall (condensation). In other words, for $s>0$, there exists mass blowing at the wall and for $s<0$, there exists mass suction.

\section{NUMERICAL SOLUTIONS AND VALIDATION}

Closed form analytical solutions of Eqns. (18)-(21) with associated boundary conditions of Eqn. (22) are extremely difficult if not intractable. A numerical procedure for solution 
of the two-point boundary value problem is therefore selected. We utilize the optimized shooting algorithm available in the symbolic code, Maple 2016 [55]. Comparison is also made with previously published results Todd [50] and Rosca and Pop [52], for local skin friction for several values of the accretion/ablation parameter $(\gamma)$ as shown in Table $\mathbf{1}$. Generally, very good correlation is achieved and confidence in the present MAPLE solutions is therefore justifiably high.

\section{RESULTS AND DISCUSSION}

Extensive numerical solutions are presented graphically in Figs. 2-11, for the influence of $\operatorname{Pr}, \gamma, N b, N t, L e, L b, P e, s$ parameters on the dimensionless velocity, temperature, nanoparticles volume fraction and microorganisms. Data has been selected where possible from existing references e.g. ([50]). We have additionally computed skin friction coefficient, heat transfer, mass transfer and microorganisms transfer characteristics with selected parameters. In the current simulations Prandtl number $(\operatorname{Pr})$ is prescribed as 6.8, (water-based nanofluid) for which it is known that gyrotactic microorganisms can thrive and remain active [56].

Figs. 2(a)-(d) illustrate the effect of the Stefan blowing parameter $(s)$ and leading edge accretion/ablation parameter $(\gamma)$ on the dimensionless velocity, temperature, nanoparticle volume fraction and motile microorganism density function profiles. The dimensionless velocity significantly increases with negative Stefan blowing parameter $(s<0)$. Generally, with suction at the $\operatorname{surface}(s=-1)$, the bionanofluid is drawn through the surface via apertures and this inhibits momentum transfer into the boundary layer by assisting 
adhesion of the boundary layer to the surface. As a result the flow is decelerated strongly and this leads to an increase in thickness of the momentum boundary layer. With stronger blowing $(s=1)$ the hot nanofluid is displaced further from the surface where the buoyancy forces accelerate the flow. This effect increases the shearing effect by increasing the maximum velocity within the boundary layer. The velocity profiles therefore are discrete for suction $(s=-1)$ and injection $(s=1)$. However, in both cases they converge smoothly to the free stream velocity indicating that a sufficiently large infinity boundary condition has been imposed in the numerical code, MAPLE. An increase in the accretion/ablation effect $(\gamma>0)$ at the leading edge manifests in a deceleration in the boundary layer flow i.e. reduction in velocities. The case of $\gamma<0$, which corresponds to backward boundary layer flow with trailing edge accretion, is not considered. Momentum boundary layer thickness is increased with $\gamma>0$. With greater suction $(s=-1)$, temperatures are reduced and with greater injection $(s=1)$ they increase. Thermal boundary layer thickness is therefore greatest with mass injection into the boundary layer. The intermediate case of $s=0$ corresponds to a solid (impermeable) wall. With increasing positive accretion/ablation rate $(\gamma>0)$ temperatures are strongly enhanced and thermal boundary layer thickness is elevated. The thermal field therefore responds very differently to leading edge accretion/ablation compared with the velocity field. With strong wall suction $(s=-1)$ the nanoparticle concentration (volume fraction) decreases continuously throughout the boundary layer. Conversely with strong blowing $(s=1)$, nano-particle concentration values are enhanced. The injection of nanofluid via the wall encourages species diffusion throughout the regime whereas removal of nanofluid inhibits species (nano-particle) diffusion. Increasing blowing therefore thickens the concentration boundary layer whereas increased suction has the 
adverse effect. Increased accretion/ablation $(\gamma>0)$ at the leading edge also exert a similar effect on nanoparticle volume fraction to that on the temperature distribution. It enhances nanoparticle concentration magnitudes consistently from the wall to the free stream. Greater injection, is also observed, to elevate microorganism density function (fig 2d) whereas stronger suction induces the reverse effect and suppresses microorganism density function. The transport of microorganisms is therefore encouraged with blowing through the wall and the corresponding boundary layer thickness is elevated. Increasing accretion/ablation also enhances microorganism density function values. In the present analysis the movement of the motile microorganisms is taken to be independent of the motion of nanoparticles. The nanoparticles are transported via Brownian motion and by not self-propulsion as with microorganisms.

Figs. 3(a)-(c) present the distribution of temperature, nanoparticle volume fraction and microorganism respectively for different values of the Brownian motion parameter $(\mathrm{Nb})$ and the suction/blowing parameter $(s)$. In these figures, again, we consider leading edge accretion/ablation to be present with $\gamma$ prescribed as $\pi / 6$. An elevation in Brownian motion parameter physically correlates with smaller nanoparticles diameters, based on the Buongiorno formulation employed in the present model. For solid wall case or injection $(s>0)$, larger $\mathrm{Nb}$ values (smaller sized nano-particles) result in enhanced thermal conduction and this in turn increase nanofluid temperatures. Conversely, smaller $\mathrm{Nb}$ values correspond to large nanoparticles which serves to inhibit thermal conduction and decreases temperatures in the nanofluid and therefore reduce the thermal boundary layer thickness. The boost in temperatures with smaller nanoparticles means that heat diffuses faster in 
nanofluids than vorticity, and this will imply a deceleration in the flow with greater Brownian motion effect, although for brevity we have omitted velocity plots here. Nanoparticle concentration (fig. 3b) is observed to be suppressed with increasing Brownian motion parameter i.e. concentration boundary layer thickness decreases with larger value of $N b$. With greater wall suction, both nanoparticle concentration (volume fraction) and also species boundary layer thickness are also decreased. The reverse effect is generated with wall blowing. The motile microorganism density function (Figure 3(c)) also decreases with increasing Brownian motion parameter. Additionally with greater wall suction, nanofluidis removed from the boundary layer regime, and this reduces the motile microorganism density. With greater wall injection, the flow is accelerated and this enhances motile microorganism density function values i.e. increases the concentration of gyrotactic micro-organisms throughout the boundary layer regime.

Figs. 4(a)-(c) depict the velocity and temperature response with the combined effects of lateral mass flux (wall suction or blowing) parameter $(s)$ and the thermophoresis parameter $(N t)$. A growth in $N t$ also induces a boost in temperatures. Thermophoretic migration of nanoparticles encourages thermal diffusion in the regime and energizes the flow. This enhances temperatures i.e. heats the nanofluid and increases thermal boundary layer thickness. Figure4 (b) also shows that a rise in nanoparticle volume fraction, $\phi(\eta)$, accompanies a larger injection. Scrutiny of figure4(c) reveals that with stronger blowing and $N t$ values, diffusion of motile microorganisms is encouraged and a substantial increase in magnitudes of motile density function produced. The converse response is computed for stronger suction. 
Figs. 5(a)-(b) present the effect of the conventional Lewis number ( $L e$ ) on nano-particle and microorganism species diffusion characteristics. Le relates the thermal diffusivity to the nanoparticle species diffusivity. When $L e=1$ the heat diffusion and nanoparticle diffusion rates are equal and thermal and nano-particle boundary layer thicknesses will be equivalent. For $L e>1$, heat diffuses faster than nanoparticle species. This reduces the efficiency of the nanoparticle migration in the nanofluid. Nanoparticle concentrations are lowest (Fig. 5(a)) with greater Lewis number and strong wall suction present. Increasing Lewis number results in a depression in microorganism density function (concentration) magnitudes. Highest values of the microorganism density function are achieved with strong injection and the lowest magnitudes correspond tostrong suction, at both values of the Lewis number.

Fig. 6 visualizes the influence of bioconvection Lewis number $(L b)$ on the dimensionless microorganism density function (concentration). Conventional Lewis number is constrained as 5 i.e. the thermal diffusion rate is five times the nanoparticle diffusion rate. For $L b$ greater that unity, the viscous diffusion rate (related to viscosity of nanofluid) exceeds motile microorganism diffusion rate. Microorganism density (concentration) is considerably enhanced with a decrease in bioconvection Lewis number. With higher microorganism diffusivity rates, the propulsion of motile microorganisms is enhanced and more even distributions through the boundary layer are results (Figure 6). In this Figure, it can be seen that wall injection always achieves higher magnitudes of micro-organism density function than wall suction. 
Fig. 7 presents the influence of the bioconvection Peclet number $(P e)$ on microorganism density function. Bioconvection Peclét number represents the ratio of advection rate of nano-particles to the diffusion rate. $P e<10$ is more appropriate for actual transport phenomena in bioconvection nanofluid mechanics (Kuznetsov 2010). Pe features only in the microorganism density conservation eqn. (21) via the coupling terms $-P e\left[\chi \phi^{\prime \prime}+\phi^{\prime} \chi^{\prime}\right]$, which effectively links the nanoparticle concentration (volume fraction), and micro-organism fields. These terms apparently have a pronounced influence on the evolution of microorganism density function in the boundary layer.In bioconvection, $P e$, when sufficiently high, is observed in practice, to significantly influence patterns of the motile microorganism flow. Bioconvection originates from the internal energy of the microorganisms. With greater swimming speed (higher bioconvection Peclet number), the microorganisms propel faster and which then reduces their concentrations i.e. density function. Injection is observed to boost microorganism concentration magnitudes whereas suction results in the reduction.

Fig. 8 and Table 2 illustrate the collective effects of leading edge accretion/ablation parameter $(\gamma)$ and wall mass flux $(s)$ parameters on wall skin friction (surface shear stress function). Increasing the leading edge accretion/ablation parametersubstantially reduces skin friction for any value of $s$. However greater values are computed when wall suction is present $(s<0)$ compared to when wall blowing is present $(s>0)$. The boundary layer flow is clearly decelerated with greater leading edge accretion/ablation effect $(\gamma>0)$. 
Fig. 9 and Table 3 show the response in wall heat transfer rate (temperature gradient) with different values of leading edge accretion/ablation parameter $(\gamma)$, Brownian motion parameter $(\mathrm{Nb})$ and wall mass flux parameter $(s)$. Increasing leading edge accretion/ablation strongly reduces the wall heat transfer rate both with wall suction and blowing. With increasing Brownian motion effect (higher $N b$ values), the nanoparticles are reduced in size. This decreases the heat transfer rate to the wall since greater temperatures are induced in the body of the fluid with smaller nanoparticles and thermal energy is retained in the fluid with lower transport rates to the wall. The opposite effect is apparent with smaller $N b$ values, which imply larger nanoparticles, lower temperatures and therefore higher heat transfer rates to the wall.

Fig. 10 and Table 4 depict the effect of leading edge accretion/ablation parameter $(\gamma)$, Lewis number $(L e)$ and wall mass flux parameter $(s)$ on nanoparticle mass transfer rate at the wall. Evidently, nanoparticle wall mass transfer rate is significantly greater with wall suction $(s<0)$ compared with wall blowing $(\mathrm{s}>0)$, implying that destruction of fluid momentum encourages nanoparticle diffusion at the wall. With greater positive values of leading edge accretion/ablation parameter, nanoparticle wall mass transfer rate, $-\phi^{\prime}(0)$ function values strongly decrease whereas with an increase in Lewis number they are markedly enhanced.

Finally, Figs. 11(a)-(b) and Tables 5-6 present the effects of different bioconvection parameters (bioconvection Lewis number and Péclet number i.e. $L b$ and $P e$ respectively) on the motile microorganism wall mass transfer rate, $-\chi^{\prime}(0)$. In Figure 11(a), increasing 
bioconvection Lewis number $(L b)$ significantly enhances the motile microorganism wall mass transfer rate irrespective of whether blowing or suction are present. With increasing leading edge accretion/ablation parameter, motile microorganism wall mass transfer rate is depressed for the case of wall injection $(s<0)$ and elevated with wall suction $(s>0)$. In Fig. 11(b), with greater bioconvection Péclet number, there is a strong increment in the motile microorganism wall mass transfer rate with either suction or injection present, although magnitudes are much reduced with larger values of accretion/ablation parameter.

\section{CONCLUSIONS}

A theoretical study has been conducted to simulate two-dimensional, unsteady, laminar, incompressible, gyrotactic bioconvection nanofluid boundary layer flow from a plane surface with leading accretion/ablation. Wall mass flux (Stefan blowing or suction) effects have also been incorporated in the model via the boundary conditions at the wall. The transformed similarity ordinary differential equations have been solved with Maple symbolic software using RKF45 quadrature with a shooting algorithm. Very close agreement with previous published solutions has been obtained. The influence of leading

edge accretion/ablation, bioconvection (bioconvection Lewis number and Péclet number), wall mass flux and nanoscale parameters on the dimensionless velocity, skin friction factor, temperature, wall heat transfer rate, nanoparticle concentration, nanoparticle wall mass transfer rate, motile micro-organism density number function and wall microorganism mass transfer rate has been studied in detail. The present computations have shown that: 
(i) An increase in the accretion/ablation effect $(\gamma>0)$ at the leading edge decelerates the boundary layer flow i.e. reduces velocity and skin friction but increases momentum boundary layer thickness.

(ii) With increasing positive accretion/ablation rate $(\gamma>0)$, temperature, nano-particle concentration (volume fraction) and microorganism density function are increased, as are the associated boundary layer thicknesses.

(iii) Temperature, nanoparticle concentration (volume fraction) and microorganism density function are decreased with stronger wall suction $(s=-1)$, and enhanced with greater wall injection $(s=1)$.

(iv) With greater bioconvection Peclét number $(\mathrm{Pe})$, microorganism density function is reduced.

(v) With larger bioconvection Lewis number $(L b)$, the motile microorganism wall mass transfer rate is enhanced.

(vi) With increasing leading edge accretion/ablation parameter, motile microorganism wall mass transfer rate is reduced for wall injection $(s<0)$ and enhanced for wall suction $(s>0)$.

(vii)With higher values of leading edge accretion/ablation parameter, nanoparticle wall mass transfer rate, is suppressed whereas with an increase in ordinary Lewis number it is elevated.

(viii) With increasing leading edge accretion/ablationparameter $(\gamma>0)$ and Brownian motion parameter $(\mathrm{Nb})$, wall heat transfer rate (Nusselt number) is decreased.

(ix) An increase in thermophoresis parameter $(N t)$ elevates both temperature and thermal boundary layer thickness. 
The present model has considered Newtonian nanofluids. Future investigations may address non-Newtonian bioconvection nanofluid flow in porous media considering gravitational forces. These will appear soon. Moreover, the considered problem can be extended for convection through square enclosure enclosing (cavity) flow [57-59].

\section{ACKNOWLEDGEMENTS}

The authors acknowledge financial support from Universiti Sains Malaysia, RU Grant 1001/PMATHS/8011013.

\section{REFERENCES}

1. Choi SUS, Eastman JA (1995) Enhancing thermal conductivity of fluids with nanoparticles. ASME Int Mech Eng Congr Expo 66:99-105.

2. Loganathan P, Vimala C (2014) Unsteady flow of nanofluids past a vertical flat plate with leading edge accretion or ablation. Indian J Phys 88:855-859. doi: $10.1007 / \mathrm{s} 12648-014-0494-6$

3. Hayat T, Imtiaz M, Alsaedi A (2016) Unsteady flow of nanofluid with double stratification and magnetohydrodynamics. Int J Heat Mass Transf 92:100-109. doi: 10.1016/j.ijheatmasstransfer.2015.08.013

4. Shehzad N, Zeeshan A, Ellahi R, Vafai K (2016) Convective heat transfer of nanofluid in a wavy channel: Buongiorno's mathematical model. J Mol Liq 222:446-455. doi: 10.1016/j.molliq.2016.07.052

5. Malvandi A, Ghasemi A, Ganji DD (2016) Thermal performance analysis of hydromagnetic $\mathrm{Al} 2 \mathrm{O} 3$-water nanofluid flows inside a concentric microannulus considering nanoparticle migration and asymmetric heating. Int $\mathrm{J}$ Therm Sci 109:10-22. doi: 10.1016/j.ijthermalsci.2016.05.023

6. Rahman MM, Alam MS, Eltayeb IA (2016) Hydromagnetic natural convective heat transfer flow in an isosceles triangular cavity filled with nanofluid using twocomponent nonhomogeneous model. Int J Therm Sci 107:272-288. doi: 10.1016/j.ijthermalsci.2016.04.009

7. Malvandi A, Moshizi SA, Ganji DD (2016) Effects of temperature-dependent thermophysical properties on nanoparticle migration at mixed convection of nanofluids in vertical microchannels. Powder Technol 303:7-19. doi: 10.1016/j.powtec.2016.08.063

8. Sheremet MA, Pop I, Roşca NC (2016) Magnetic field effect on the unsteady 
natural convection in a wavy-walled cavity filled with a nanofluid: Buongiorno's mathematical model. J Taiwan Inst Chem Eng 61:211-222. doi: 10.1016/j.jtice.2015.12.015

9. Akilu S, Sharma K V, Baheta AT, Mamat R (2016) A review of thermophysical properties of water based composite nanofluids. Renew Sustain Energy Rev 66:654-678. doi: 10.1016/j.rser.2016.08.036

10. Ilhan B, Kurt M, Ertürk H (2016) Experimental investigation of heat transfer enhancement and viscosity change of hBN nanofluids. 77:272-283. doi: 10.1016/j.expthermflusci.2016.04.024

11. Agarwal R, Verma K, Kumar N, Singh R (2017) Sensitivity of thermal conductivity for Al 2 O 3 nanofluids. Exp Therm Fluid Sci 80:19-26. doi: 10.1016/j.expthermflusci.2016.08.007

12. Loganathan P, Chand PN, Ganesan P (2013) Radiation effects on an unsteady natural convective flow of a nanofluid past an infinite verticle plate. 8:1-10. doi: 10.1142/S179329201350001X

13. Mejri I, Mahmoudi A, Abbassi MA, Omri A (2014) Magnetic field effect on entropy generation in a nanofluid-filled enclosure with sinusoidal heating on both side walls. Powder Technol 266:340-353. doi: 10.1016/j.powtec.2014.06.054

14. Uddin MJ, Khan WA, Ismail AIM (2012) Lie group analysis of natural convective flow from a convectively heated upward facing radiating permeable horizontal plate in porous media filled with nanofluid. J Appl Math 2012:18 pages. doi: $10.1155 / 2012 / 648675$

15. Hatami M, Sheikholeslami M, Ganji DD (2014) Laminar flow and heat transfer of nanofluid between contracting and rotating disks by least square method. Powder Technol 253:769-779. doi: 10.1016/j.powtec.2013.12.053

16. Freidoonimehr N, Rashidi MM, Mahmud S (2015) Unsteady MHD free convective flow past a permeable stretching vertical surface in a nano-fluid. Int $\mathbf{J}$ Therm Sci 87:136-145. doi: 10.1016/j.ijthermalsci.2014.08.009

17. Beg OA, Rashidi MM, Akbari M, Hosseini A (2014) Comparative Numerical Study of Single-Phase and Two-Phase Models for Bio-Nanofluid Transport Phenomena. J Mech Med Biol 14:1450011. doi: 10.1142/S0219519414500110

18. Mutuku WN, Makinde OD (2014) Hydromagnetic bioconvection of nanofluid over a permeable vertical plate due to gyrotactic microorganisms. Comput Fluids 95:8897. doi: 10.1016/j.compfluid.2014.02.026

19. Siddiqa S, Gul-e-Hina, Begum N, et al (2016) Numerical solutions of nanofluid bioconvection due to gyrotactic microorganisms along a vertical wavy cone. Int $\mathbf{J}$ Heat Mass Transf 101:608-613. doi: 10.1016/j.ijheatmasstransfer.2016.05.076

20. Acharya N, Das K, Kumar P (2016) Framing the effects of solar radiation on magneto-hydrodynamics bioconvection nano fl uid fl ow in presence of gyrotactic microorganisms. J Mol Liq 222:28-37. doi: 10.1016/j.molliq.2016.07.023 
21. Kuznetsov AV (2006) The onset of thermo-bioconvection in a shallow fluid saturated porous layer heated from below in a suspension of oxytactic microorganisms. Eur $\mathrm{J}$ Mech B/Fluids 25:223-233. doi: 10.1016/j.euromechflu.2005.06.003

22. Geng P, Kuznetsov AV (2004) Effect of small solid particles on the development of bioconvection plumes. Int Commun Heat Mass Transf 31:629-638. doi: 10.1016/S0735-1933(04)00050-8

23. Kuznetsov AV, Nield DA (2014) Natural convective boundary-layer flow of a nanofluid past a vertical plate: A revised model. Int J Therm Sci 77:126-129. doi: 10.1016/j.ijthermalsci.2013.10.007

24. Kuznetsov AV (2012) Nanofluid Bioconvection in a Horizontal Fluid-Saturated Porous Layer. J Porous Media 15:11-27.

25. Kuznetsov AV (2011) Bio-thermal convection induced by two different species of microorganisms. Int Commun Heat Mass Transf 38:548-553. doi: 10.1016/j.icheatmasstransfer.2011.02.006

26. Kuznetsov AV (2005) Thermo-bioconvection in a suspension of oxytactic bacteria. Int Commun Heat Mass Transf 32:991-999. doi: 10.1016/j.icheatmasstransfer.2004.11.005

27. Kuznetsov AV, Avramenko AA, Geng P (2004) Analytical investigation of a falling plume caused by bioconvection of oxytactic bacteria in a fluid saturated porous medium. Int J Eng Sci 42:557-569. doi: 10.1016/j.ijengsci.2003.08.004

28. Makinde OD, Animasaun IL (2016) Thermophoresis and Brownian motion effects on MHD bioconvection of nano fl uid with nonlinear thermal radiation and quartic chemical reaction past an upper horizontal surface of a paraboloid of revolution. $\mathbf{J}$ Mol Liq 221:733-743. doi: 10.1016/j.molliq.2016.06.047

29. Akbar NS, Khan ZH (2016) Magnetic field analysis in a suspension of gyrotactic microorganisms and nanoparticles over a stretching surface. J Magn Magn Mater 410:72-80. doi: 10.1016/j.jmmm.2016.02.075

30. Amirsom NA, Uddin MJ, Ismail AI (2016) Three dimensional stagnation point flow of bionanofluid with variable transport properties. Alexandria Eng J 55:19831993. doi: 10.1016/j.aej.2016.07.016

31. Jayachandra Babu M, Sandeep N (2016) Effect of nonlinear thermal radiation on non-aligned bio-convective stagnation point flow of a magnetic-nanofluid over a stretching sheet. Alexandria Eng J 55:1931-1939. doi: 10.1016/j.aej.2016.08.001

32. Raees A, Raees-ul-Haq M, Xu H, Sun Q (2016) Three-dimensional stagnation flow of a nanofluid containing both nanoparticles and microorganisms on a moving surface with anisotropic slip. Appl Math Model 40:4136-4150. doi: 10.1016/j.apm.2015.11.022

33. Fang T, Jing W (2014) Flow, heat, and species transfer over a stretching plate considering coupled Stefan blowing effects from species transfer. Commun 
Nonlinear Sci Numer Simul 19:3086-3097. doi: 10.1016/j.cnsns.2014.02.009

34. Nellis G, Klein S (2008) Heat Transfer. Cambridge university press

35. J.H. Lienhard, IV And J.H. Lienhard V (2005) A Heat Transfer Text Book, 3rd ed. Phlogiston Press, U.S.A.

36. Uddin J, Kabir MN, Bég OA (2016) Computational investigation of Stefan blowing and multiple-slip effects on buoyancy-driven bioconvection nanofluid flow with microorganisms. Int $\mathrm{J}$ Heat Mass Transf 95:116-130. doi: 10.1016/j.ijheatmasstransfer.2015.11.015

37. Buongiorno J (2006) Convective Transport in Nanofluids. J Heat Transfer 128:240. doi: $10.1115 / 1.2150834$

38. Garoosi F, Garoosi S, Hooman K (2014) Numerical simulation of natural convection and mixed convection of the nano fl uid in a square cavity using Buongiorno model. Powder Technol 268:279-292. doi: 10.1016/j.powtec.2014.08.006

39. Garoosi F, Jahanshaloo L, Garoosi S (2015) Numerical simulation of mixed convection of the nano fl uid in heat exchangers using a Buongiorno model. Powder Technol 269:296-311. doi: 10.1016/j.powtec.2014.09.009

40. Malvandi A, Moshizi SA, Ghadam E, Ganji DD (2014) Modified Buongiorno ' s model for fully developed mixed convection flow of nanofluids in a vertical annular pipe. Comput Fluids 89:124-132. doi: 10.1016/j.compfluid.2013.10.040

41. Garoosi F, Jahanshaloo L, Mehdi M, et al (2015) Numerical simulation of natural convection of the nanofluid in heat exchangers using a Buongiorno model. Appl Math Comput 254:183-203. doi: 10.1016/j.amc.2014.12.116

42. Sheremet MA, Pop I (2015) Mixed convection in a lid-driven square cavity filled by a nanofluid : Buongiorno 's mathematical model. Appl Math Comput 266:792808. doi: 10.1016/j.amc.2015.05.145

43. Sheremet MA, Pop I, Shenoy A (2015) Unsteady free convection in a porous open wavy cavity filled with a nanofluid using Buongiorno's mathematical model. Int Commun Heat Mass Transf 67:66-72. doi: 10.1016/j.icheatmasstransfer.2015.07.007

44. Tham L, Nazar R, Pop I (2014) Mixed convection flow from a horizontal circular cylinder embedded in a porous medium filled by a nanofluid: Buongiorno-Darcy model. Int J Therm Sci 84:21-33. doi: 10.1016/j.ijthermalsci.2014.04.020

45. Sheremet MA, Pop I (2014) Conjugate natural convection in a square porous cavity filled by a nanofluid using Buongiorno's mathematical model. Int J Heat Mass Transf 79:137-145. doi: 10.1016/j.ijheatmasstransfer.2014.07.092

46. Sheremet MA, Pop I (2015) Free convection in a triangular cavity filled with a porous medium saturated by a nanofluid. Int $\mathbf{J}$ Numer Methods Heat Fluid Flow 25:1138-1161. doi: 10.1108/HFF-06-2014-0181 
47. Noghrehabadi A, Behseresht A, Ghalambaz M, Behseresht J (2013) NaturalConvection Flow of Nanofluids Over Vertical Cone Embedded in Non-Darcy Porous Media. J Thermophys Heat Transf 27:334-341. doi: 10.2514/1.T3965

48. Rashidi MM, Momoniat E, Ferdows M, Basiriparsa A (2014) Lie Group Solution for Free Convective Flow of a Nanofluid Past a Chemically Reacting Horizontal Plate in a Porous Media. Math Probl Eng 2014:1-21. doi: 10.1155/2014/239082

49. Garoosi F, Jahanshaloo L, Rashidi MM, et al (2015) Numerical simulation of natural convection of the nanofluid in heat exchangers using a Buongiorno model. Appl Math Comput 254:183-203. doi: 10.1016/j.amc.2014.12.116

50. Todd L (1997) A family of laminar boundary layers along a semi-infinite flat plate. Fluid Dyamics Reasearch 19:235-249.

51. Fang $T$ (2008) International Journal of Non-Linear Mechanics A note on the unsteady boundary layers over a flat plate. 43:1007-1011. doi: 10.1016/j.ijnonlinmec.2008.06.010

52. Rosca NC, Pop I (2014) Unsteady boundary layer flow of a nanofluid past a moving surface in an external uniform free stream using Buongiorno's model. Comput Fluids 95:49-55. doi: 10.1016/j.compfluid.2014.02.011

53. Kuznetsov AV (2010) The onset of nanofluid bioconvection in a suspension containing both nanoparticles and gyrotactic microorganisms. Int Commun Heat Mass Transf 37:1421-1425. doi: 10.1016/j.icheatmasstransfer.2010.08.015

54. Stewartson K (2008) On the motion of a flat plate at high speed in a viscous compressible fluid. I. Impulsive motion. Math Proc Cambridge Philos Soc 51:202. doi: $10.1017 / \mathrm{S} 0305004100030061$

55. Aziz A, Khan WA, Pop I (2012) Free convection boundary layer flow past a horizontal flat plate embedded in porous medium filled by nanofluid containing gyrotactic microorganisms. Int $\mathrm{J}$ Therm Sci 56:48-57. doi: 10.1016/j.ijthermalsci.2012.01.011

56. Anoop KB, Sundararajan T, Das SK (2009) Effect of particle size on the convective heat transfer in nanofluid in the developing region. Int $\mathrm{J}$ Heat Mass Transf 52:2189-2195. doi: 10.1016/j.ijheatmasstransfer.2007.11.063

57. Javed T, Mehmood Z, Abbas Z (2017) Natural convection in square cavity filled with ferrofluid saturated porous medium in the presence of uniform magnetic field. Phys B Condens Matter 506:122-132. doi: 10.1016/j.physb.2016.11.008

58. Javed T, Mehmood Z, Siddiqui MA, Pop I (2017) Effects of uniform magnetic field on the natural convection of $\mathrm{Cu}$-water nanofluid in a triangular cavity. Int J Numer Methods Heat Fluid Flow 27:334-357. doi: 10.1108/HFF-10-2015-0448

59. Javed T, Siddiqui MA, Mehmood Z, Pop I (2015) MHD natural convective flow in an isosceles triangular cavity filled with porous medium due to uniform/nonuniform heated side walls. Zeitschrift fur Naturforsch - Sect A J Phys Sci 70:919928. doi: 10.1515/zna-2015-0232 


\section{FIGURES}

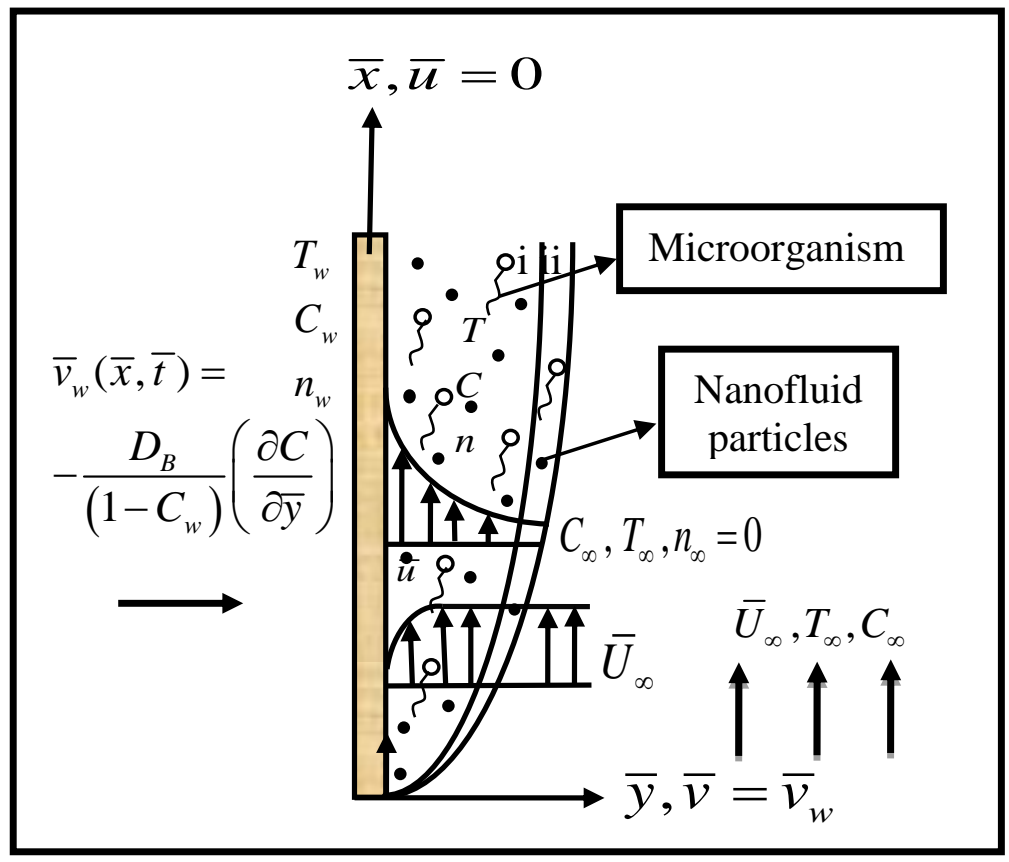

Figure 1: Flow model and coordinate system
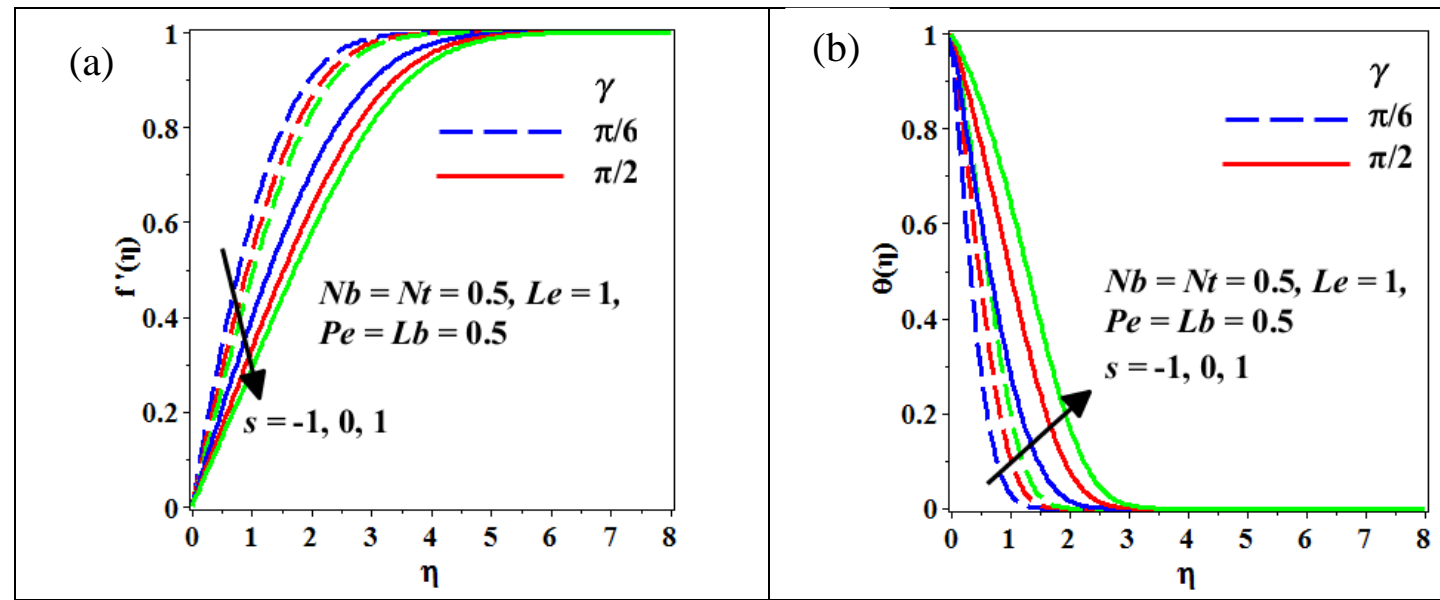

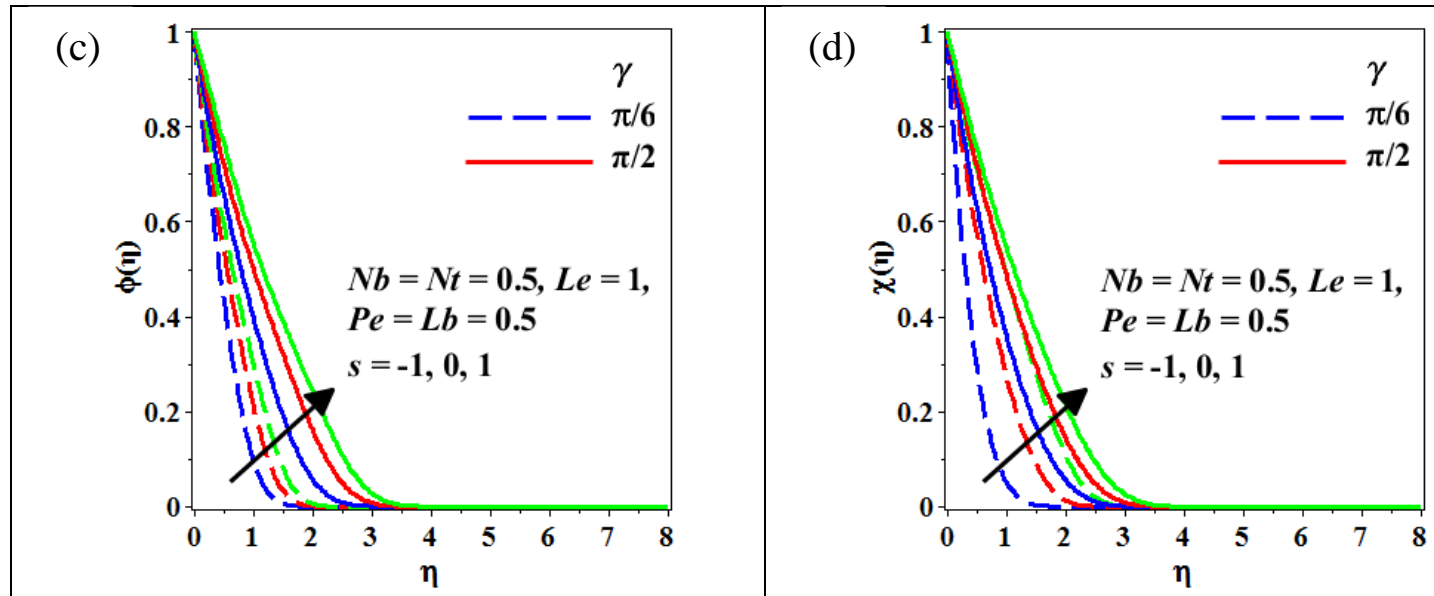

Figure 2. Variation of $f^{\prime}(\eta), \theta(\eta), \phi(\eta)$ and $\chi(\eta)$ with different values of $\gamma$ and $s$

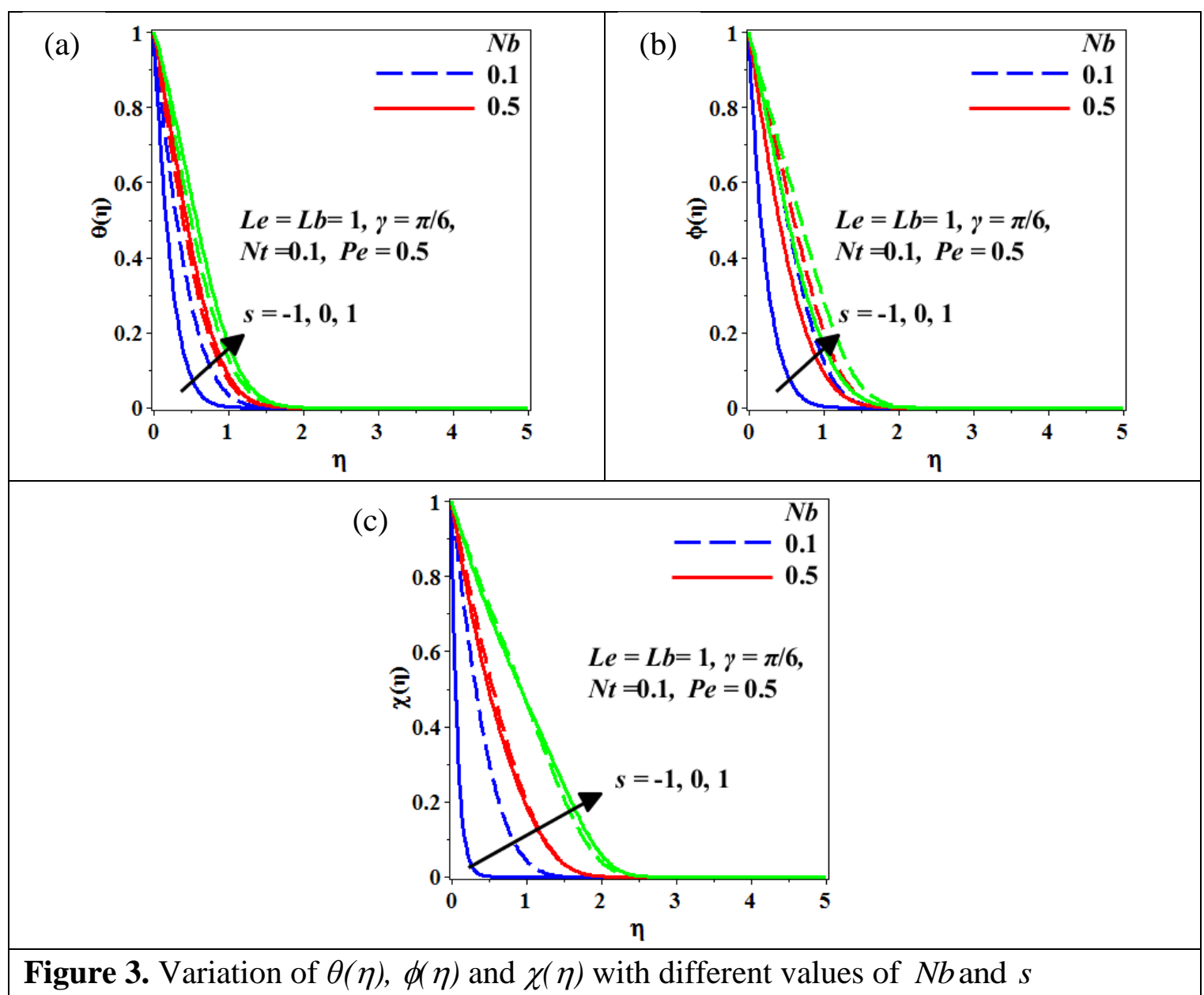



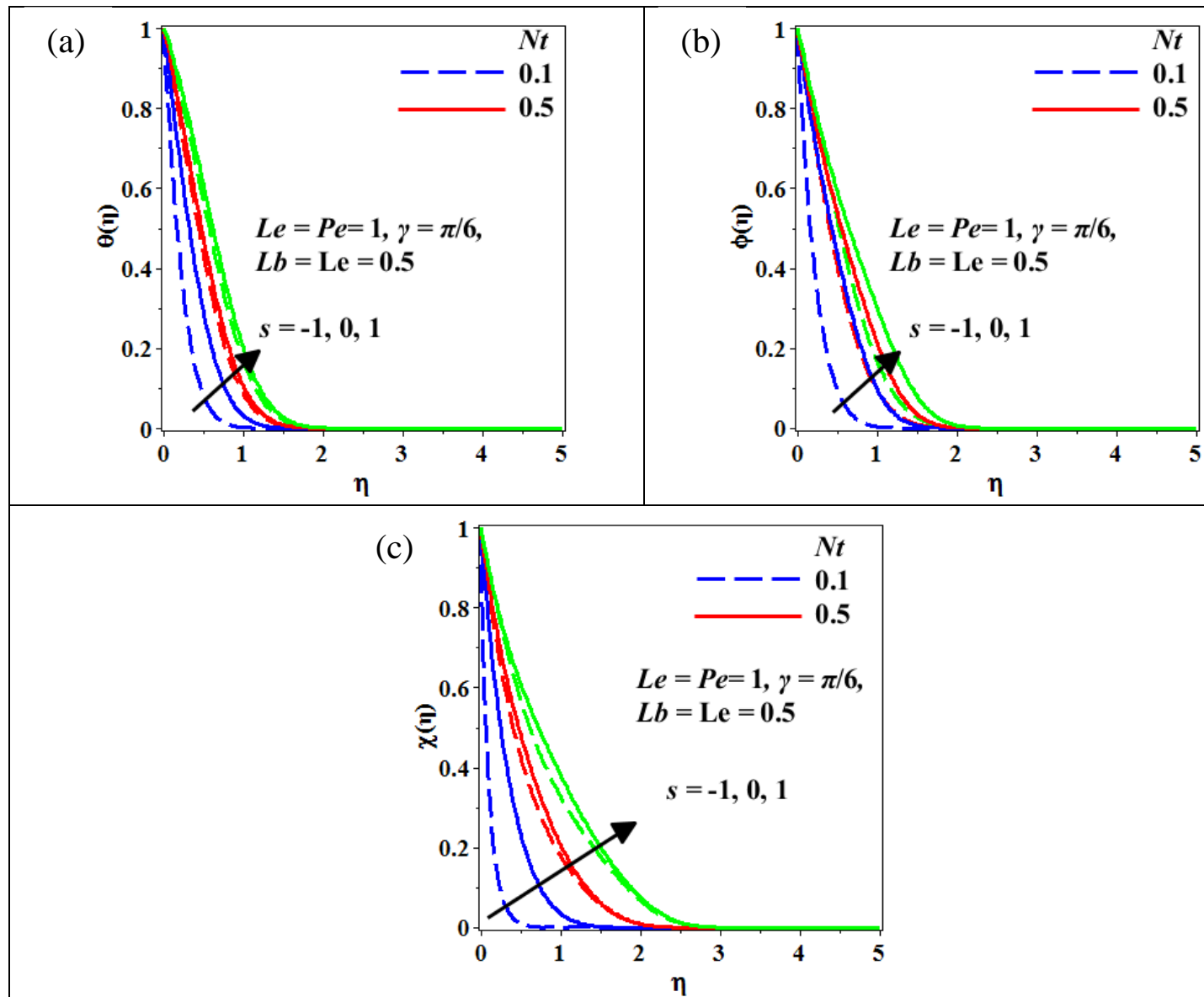

Figure 4. Variation of $\theta(\eta), \phi(\eta)$ and $\chi(\eta)$ with different values of $N t$ and $s$
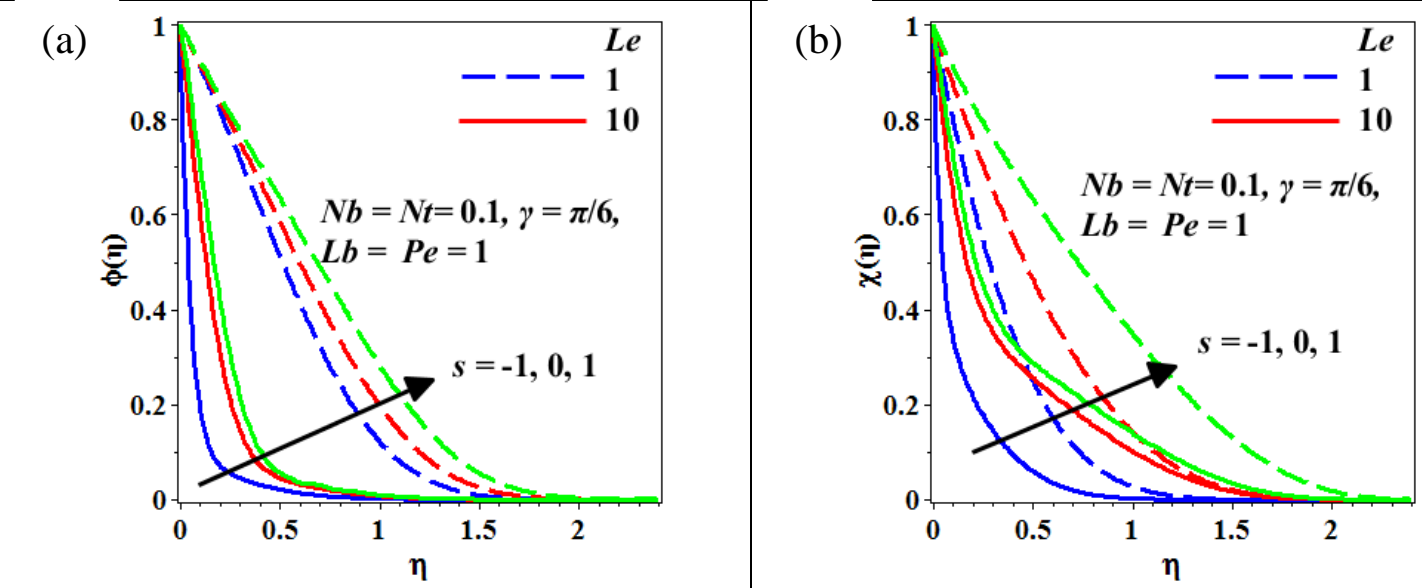

Figure 5. Variation of $\phi(\eta)$ and $\chi(\eta)$ with different values of Le and $s$ 


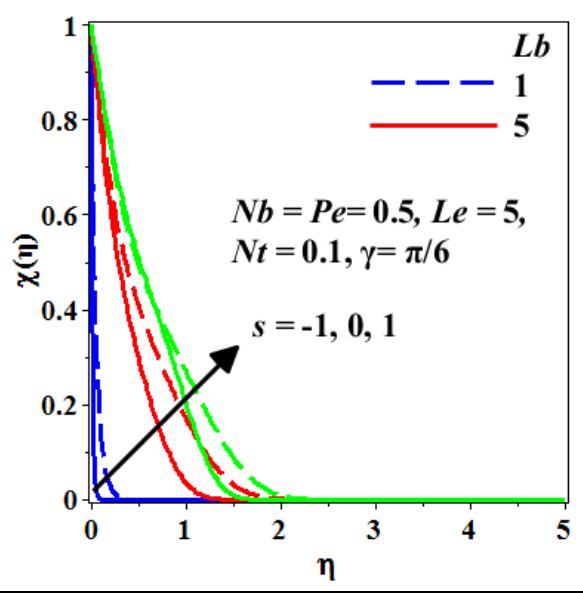

Figure 6. Variation of $\chi(\eta)$ with different values of $L b$ and $s$

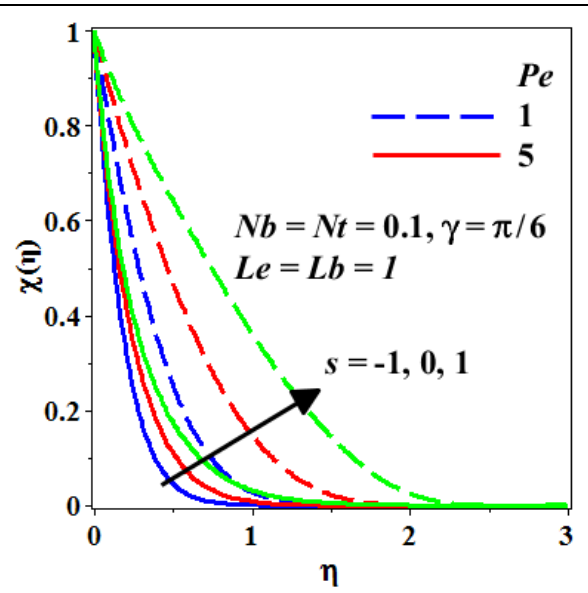

Figure 7. Variation of $\chi(\eta)$ with different values of $P e$ and $s$

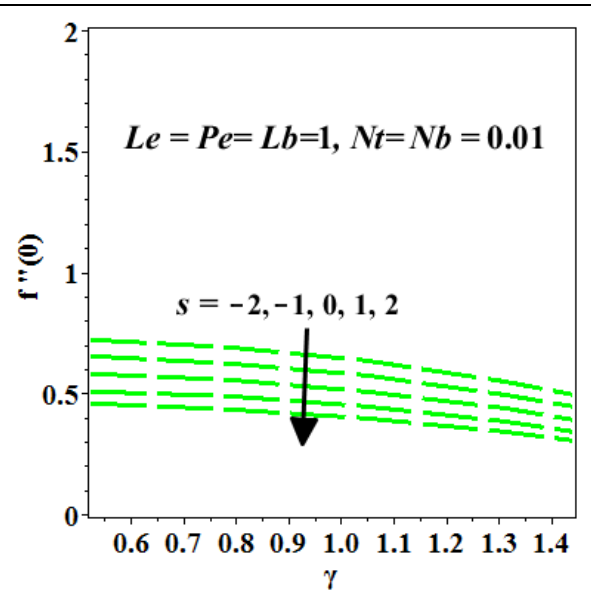

Figure 8. Effect of $s$ on $f^{\prime \prime}(0)$ with different values of $\gamma$ 

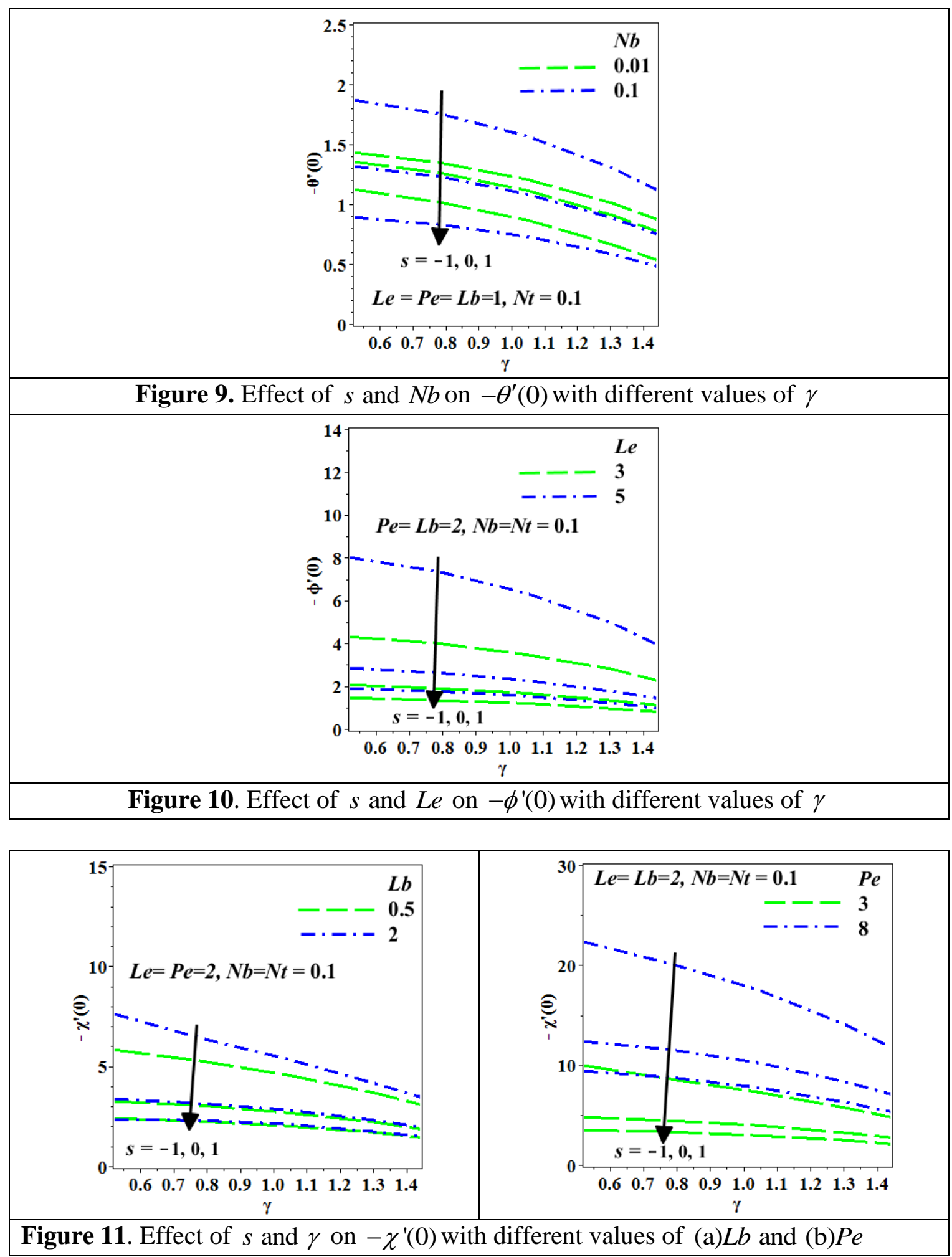


\section{TABLES}

Table 2: Values of $f^{\prime \prime}(0)$ when $N t=N b=0.01, L b=P e=L e=1$.

\begin{tabular}{|c|c|c|c|c|c|}
\hline \multirow{2}{*}{$\gamma$} & \multicolumn{5}{|c|}{$f^{\prime \prime}(0)$} \\
\cline { 2 - 6 } & -2 & -1 & 0 & 1 & 2 \\
\hline$\pi / 6$ & 0.720557 & 0.650015 & 0.577003 & 0.508971 & 0.454212 \\
\hline$\pi / 4$ & 0.691224 & 0.623326 & 0.552876 & 0.487366 & 0.434987 \\
\hline$\pi / 3$ & 0.635321 & 0.572555 & 0.507223 & 0.446679 & 0.398731 \\
\hline $5 \pi / 12$ & 0.549287 & 0.494352 & 0.436897 & 0.384044 & 0.342919 \\
\hline $11 \pi / 24$ & 0.492358 & 0.442474 & 0.390113 & 0.342354 & 0.305825 \\
\hline
\end{tabular}

Table 3: Values of $-\theta^{\prime}(0)$ when $N t=0.1, L b=P e=L e=1$.

\begin{tabular}{|c|c|c|c|c|c|c|}
\hline \multirow{2}{*}{$\gamma$} & \multicolumn{5}{|c|}{$-\theta^{\prime}(0)$} \\
\cline { 2 - 7 } & \multicolumn{3}{|c|}{$N b=0.01$} & \multicolumn{3}{|c|}{$N b=0.1$} \\
\cline { 2 - 7 } & $s=-1$ & $s=0$ & $s=1$ & $s=-1$ & $s=0$ & $s=1$ \\
\hline$\pi / 6$ & 1.425487 & 1.349263 & 1.119020 & 1.868920 & 1.315193 & 0.893898 \\
\hline$\pi / 4$ & 1.342286 & 1.258437 & 1.014321 & 1.754277 & 1.226549 & 0.827925 \\
\hline$\pi / 3$ & 1.205121 & 1.114820 & 0.864940 & 1.567688 & 1.086215 & 0.726301 \\
\hline $5 \pi / 12$ & 1.005496 & 0.908305 & 0.661985 & 1.295692 & 0.883449 & 0.581199 \\
\hline $11 \pi / 24$ & 0.876657 & 0.774474 & 0.535403 & 1.117525 & 0.750455 & 0.486465 \\
\hline
\end{tabular}

Table 4: Values of $-\phi^{\prime}(0)$ when $N b=N t=0.1, L b=P e=2$.

\begin{tabular}{|c|c|c|c|c|c|c|}
\hline \multirow{2}{*}{$\gamma$} & \multicolumn{5}{|c|}{$L e=3$} & \multicolumn{5}{|c|}{$\phi^{\prime}(0)$} \\
\cline { 2 - 7 } & \multicolumn{3}{|c|}{$s e=5$} \\
\cline { 2 - 7 } & $s=-1$ & $s=0$ & $s=1$ & $s=-1$ & $s=0$ & $s=1$ \\
\hline$\pi / 4$ & 4.029639 & 1.923253 & 1.356700 & 7.452991 & 2.659292 & 1.791047 \\
\hline$\pi / 3$ & 3.523624 & 1.685283 & 1.190551 & 6.442987 & 2.308076 & 1.557556 \\
\hline $5 \pi / 12$ & 2.795667 & 1.343835 & 0.952118 & 4.991364 & 1.805328 & 1.223204 \\
\hline $11 \pi / 24$ & 2.313666 & 1.119396 & 0.795655 & 4.017437 & 1.472500 & 0.388818 \\
\hline
\end{tabular}


Table 5: Values of $-\chi^{\prime}(0)$ when $N t=N b=0.1, P e=L e=2$.

\begin{tabular}{|c|c|c|c|c|c|c|}
\hline \multirow{2}{*}{$\gamma$} & \multicolumn{5}{|c|}{$-\chi^{\prime}(0)$} \\
\cline { 2 - 7 } & \multicolumn{3}{|c|}{$L b=0.5$} & \multicolumn{3}{c|}{$L b=2$} \\
\cline { 2 - 7 } & -1 & 0 & 1 & -1 & 0 & 1 \\
\hline$\pi / 6$ & 5.840798 & 3.232983 & 2.385871 & 7.621526 & 3.351096 & 2.341084 \\
\hline$\pi / 4$ & 5.252215 & 3.023618 & 2.261013 & 6.398876 & 3.138006 & 2.291537 \\
\hline$\pi / 3$ & 4.562483 & 2.689649 & 2.027465 & 5.328916 & 2.797387 & 2.089943 \\
\hline $5 \pi / 12$ & 3.665373 & 2.204979 & 1.674657 & 4.145867 & 2.301754 & 1.750409 \\
\hline $11 \pi / 24$ & 3.098377 & 1.886119 & 1.439424 & 3.451491 & 1.974589 & 1.515804 \\
\hline
\end{tabular}

Table 6: Values of

\begin{tabular}{|c|c|c|c|c|c|c|}
\hline \multirow{2}{*}{$\gamma$} & \multicolumn{6}{|c|}{$-\chi^{\prime}(0)$} \\
\cline { 2 - 7 } & \multicolumn{3}{|c|}{$P e=3$} & \multicolumn{3}{c|}{$P e=8$} \\
\cline { 2 - 7 } & -1 & 0 & 1 & -1 & 0 & 1 \\
\hline$\pi / 6$ & 9.986980 & 4.766449 & 3.480706 & 22.302116 & 12.342187 & 9.435950 \\
\hline$\pi / 4$ & 8.607940 & 4.453843 & 3.313131 & 20.132746 & 11.515440 & 8.756513 \\
\hline$\pi / 3$ & 7.282920 & 3.956611 & 2.972202 & 17.504888 & 10.203930 & 7.739319 \\
\hline $5 \pi / 12$ & 5.726681 & 3.235709 & 2.450701 & 14.037149 & 8.306593 & 6.293144 \\
\hline $11 \pi / 24$ & 4.785869 & 2.761285 & 2.101388 & 11.832668 & 7.060678 & 5.349604 \\
\hline
\end{tabular}

